\title{
Boundary Conforming Delaunay Mesh Generation
}

\author{
Hang Si* \\ *Weierstrass Institute, Berlin, si@wias-berlin.de
}

February 29, 2008

Many physical phenomena can be modeled by partial differential equations which may be solved by numerical methods such as finite element and finite volume methods. In this context, a given domain must first be subdivided into many simple cells. Both the shape and size of the cells will affect the accuracy and convergence of the method. A boundary conforming Delaunay mesh is a partition of a polyhedral domain into simplices that satisfy the Delaunay and additional criteria. It has many optimal properties and is particularly needed in Voronoi-box-based finite volume schemes [1] which are well-suited for diffusion and transportation problems, such as, porous media flow, semiconduct device simulation, etc. In this paper, we discuss the problem of generating three-dimensional boundary conforming Delaunay meshes with well-shaped tetrahedra and appropriate mesh size.

First of all, we approximate the physical domain $\Omega$ as well as its boundary $\partial \Omega$ by a piecewise linear system (abbreviated as PLS) which is the generalization of the geometric object introduced by Miller et al. [3]. Simply saying, an $n$-dimensional PLS $\mathcal{X}$ is a collection of $i$-polytopes $(-1 \leq i \leq n)$. Each $i$-polytope is a union of $i$-dimensional convex polytopes which have the same $i$-dimensional affine hull. The polytopes in $\mathcal{X}$ are closed under taking boundaries. The intersection of any two polytopes of $\mathcal{X}$ is again a polytope of $\mathcal{X}$ (note that $\emptyset \in \mathcal{X}$ ). The underlying space of $\mathcal{X},|\mathcal{X}|$, is the union of all polytopes of $\mathcal{X}$. The $i$-skeleton of $\mathcal{X}, \mathcal{X}^{(i)}$, is the collection of polytopes of $\mathcal{X}$ with dimension $\leq i$. Hence, $\Omega \subset \mathbb{R}^{n}$ and $\partial \Omega$ can be approximated (topologically and geometrically) by $|\mathcal{X}|$ and $\left|\mathcal{X}^{(n-1)}\right|$, respectively.

A boundary conforming Delaunay mesh of $\mathcal{X}$ is a simplicial complex $\mathcal{T}$ (of dimension $n$ ) such that (i) $|\mathcal{X}|=|\mathcal{T}|$, (ii) $\left|\mathcal{X}^{(n-1)}\right|=|\mathcal{K}|$, where $\mathcal{K}$ is a subcomplex of $\mathcal{T}$, (iii) every simplex of $\mathcal{T}$ is Delaunay, and (iv) any simplex $\sigma \in \mathcal{K}$ has its smallest circumscribed sphere $\Sigma$ empty, i.e., $\Sigma$ contains no vertex of $\mathcal{T}$. The dual of a boundary conforming Delaunay mesh is just the right Voronoi partition for the Voronoi-box-based finite volume scheme.

We then study a classical meshing technique, called Delaunay refinement, 
for generating three-dimensional boundary conforming Delaunay mesh with guaranteed well-shaped tetrahedra. In particular, Shewchuk's algorithm [4] is reanalyzed. We show that this algorithm terminates for a larger class of inputs than previously proven. Let $\mathcal{X}$ be a three-dimensional PLS, and $\theta_{m}$ be the smallest input angle between adjacent polytopes of $\mathcal{X}$. We prove that the algorithm always terminates as long as $\theta_{m} \geq \arccos \frac{1}{2 \sqrt{2}} \approx 69.3^{\circ}$ (which was $\theta_{m} \geq 90^{\circ}$ in [4]). Moreover, the restriction on the smallest planar angle (which was $60^{\circ}$ in [4]) can be removed by a minor modification in the original algorithm. This enables the generation of boundary conforming Delaunay meshes from surface meshes which may contain small planar angles but no small dihedral angle $\left(<69.3^{\circ}\right)$.

It is observed that the change of point insertion order will result various sizes in the outputs. We investigated several point insertion orders. The output mesh size with a specific insertion order is analyzed. With a modest assumption on the inputs, a polylog $(\Delta)$ upper bound on the output mesh size is derived, where $\Delta$ is the spread of the input - the ratio of the longest to the shortest pairwise distance among input points. No previous analysis provided bound on output mesh size depending only on the input.

Delaunay refinement will generate well-shaped tetrahedra except slivers which are very flat tetrahedra. We test the use of Delaunay refinement to remove slivers and compare the results with a theoretical guaranteed sliverfree algorithm [2]. A tetrahedron in the mesh is identified as a sliver if its smallest dihedral angle $<5^{\circ}$. For PLSs having no small input angles, the method successful removes all slivers, and the number of added points is $O\left(n_{s}\right)$, where $n_{s}$ is the number of initial slivers. This bound is much smaller than the one given in [2].

\section{References}

[1] R. Eymard, T. Gallouet, and R. Herbin. The finite volume method. In P. G. Ciarlet and J. L. Lions, editors, Handbook of Numerical Analysis, volume VII, pages 715-1022. North-Holland, Amsterdam, 2000.

[2] X.-Y. Li and S.-H. Teng. Generating well-shaped Delaunay meshes in 3D. In Proc. 12th annual ACM-SIAM Symposium on Discrete Algorithms, pages 28-37, Washington, DC, USA, 2001.

[3] G. L. Miller, D. Talmor, S.-H. Teng, N. J. Walkington, and H. Wang. Control volume meshes using sphere packing: Generation, refinement and coarsening. In Proc. 5th International Meshing Roundtable. Sandia National Laboratories, 1996.

[4] J. R. Shewchuk. Delaunay Refinement Mesh Generation. PhD thesis, Department of Computer Science, Carnegie Mellon University, Pittsburgh, Pennsylvania, 1997. Available as Technical Report CMU-CS-97-137. 